\title{
CONTINUITY OF EXCESSIVE HARMONIC FUNCTIONS FOR CERTAIN DIFFUSIONS
}

\author{
Z. R. POP-STOJANOVIĆ
}

(Communicated by Bert E. Fristedt)

\begin{abstract}
This paper shows that under certain analytic conditions concerning its potential kernel, a strong Markov process, which is transient and with continuous sample paths, has all of its excessive harmonic functions, which are not identically infinite, continuous, Harnack inequality holds, and a family of its excessive functions is equi-continuous on compacts.
\end{abstract}

Introduction. In this paper we are dealing with a strong Markov process with continuous sample paths satisfying analytic conditions which are slightly stronger than those introduced in earlier works of K. L. Chung and Murali Rao [2, 3]. These conditions concern the potential kernel of the process, and neither duality assumptions nor any assumptions concerning $\alpha$-potential kernel, for $\alpha>0$, are needed here. Under these additional conditions concerning the potential kernel of the process we are able to show that all harmonic functions of the process are continuous-not just locally bounded ones. This is the main reason why we are using a direct approach in dealing with harmonic functions here. It can be shown that under our setting we have a $P$-harmonic space in the sense of Constantinescu and Cornea [4]; although some results such as continuity of locally bounded harmonic functions do follow from $P$-harmonic space structure, it appears that the continuity of all harmonic functions is not derived anywhere in the literature as a consequence of $P$ harmonic space structure. Also, it is possible to transform a "nice" potential kernel into a "nice" potential kernel while preserving the structure of a $P$-harmonic space as in cases of killing and time changes. However, the effect of this transformation on general harmonic functions of the process is not easily determined. Having all these in mind, we will use the following direct approach in dealing with harmonic functions of a diffusion process considered in this paper.

SETTING. (i) Throughout this paper $X=\left(\Omega, \mathscr{F}, \mathscr{F}_{t}, X_{t}, \theta_{t}, P^{x}\right)$ will denote a transient diffusion, i.e., a strong Markov process with continuous sample paths on a locally compact Hausdorff state space $(E, \mathscr{E})$ with a countable base. Here, the transience means that for every compact set $K$ and every $x$,

$$
\lim _{t \rightarrow \infty} P^{x}\left[T_{K} \cdot \theta_{t}<\infty\right]=0 .
$$

(Notations throughout this paper are generally that of Blumenthal and Getoor [1].)

Received by the editors March 27, 1987 and, in revised form, April 27, 1987.

1980 Mathematics Subject Classification (1985 Revision). Primary 60J45, 60J25; Secondary $31 \mathrm{C} 05$.

Key words and phrases. Strong Markov process; transience; excessive harmonic functions; Harnack inequality. 
(ii) Following [2, 3], we are assuming the existence of a potential kernel with following properties.

Let

$$
U(x, d y)=u(x, y) \xi(d y)
$$

denote this kernel where $\xi$ is a Radon measure, and the potential density function $u$ is such that:

(a) For every $x$, and for every $y$, function $(x, y) \rightarrow u^{-1}(x, y)$ is finite and continuous; in particular, this implies $u(x, y)>0$ for all $(x, y)$.

(b) $u(x, y)=\infty$ if and only if $x=y$.

REMARK. A weaker assumption such as $\left.u\right|_{K \times L}$ continuous for all pairs $(K, L)$ of disjoint compacts will suffice for all arguments in this paper. However, in forthcoming papers which are sequel to this (a) will be needed.

Recall that

(c) For every nonnegative measurable function $f$,

$$
E^{x}\left[\int_{0}^{\infty} f\left(X_{t}\right) d t\right]=\int u(x, y) f(y) \xi(d y) .
$$

Other notations used here are: if $\left(P_{t}\right)$ denotes the transition semigroup of the process $X$, then:

$$
\begin{aligned}
P_{t} u(x, y) & =\int P_{t}(x, d z) u(z, y) \\
P_{A} u(x, y) & =\int P_{A}(x, d z) u(z, y),
\end{aligned}
$$

where $A$ is a Borel set in $\mathscr{E}$;

$$
P_{A} f(x)=E^{x}\left[f\left(X_{T_{A}}\right) ; T_{A}<+\infty\right],
$$

where

$$
T_{A}=\inf \left\{t>0 ; X_{t} \in A\right\}
$$

denotes the hitting time of the set $A$; here, $f$ is a Borel measurable function. Let us recall the following:

A function $s$ is superaveraging if for every $t, s \geq P_{t} s$.

The excessive regularization of $s$ is denoted by $\underline{s}$, i.e.,

$$
\underline{s}=\lim _{t \rightarrow 0} P_{t} s .
$$

A superaveraging function $s$ is excessive if $s=\underline{s}$.

In dealing with a potential density it has been proved in [2] that starting from a version $v(x, y)$ of $U(x, d y) / \xi(d y)$ such that $v(\cdot, y)$ is excessive and finite $\xi$-a.e. for every $y$, and assuming points are polar, then a "round" version $w(x, y)$ of $U(x, d y) / \xi(d y)$ can be provided: $w(\cdot, y)$ is excessive for all $y$, and

$$
P_{G} w(x, y)=w(x, y)
$$

for every $x \in E$, for every $y \in G$ and for every open set $G$. Also, it follows that for every $y$, one has

$$
w(\cdot, y) \equiv\left\{\begin{array}{c}
u(\cdot, y) \\
0
\end{array}\right.
$$

This leads to the following exceptional set. 
DEFINITION. Let

$$
Z=\{y ; w(\cdot, y) \equiv 0\}=\{y ; w(\cdot, y) \not \equiv u(\cdot, y)\} .
$$

It has been shown [2] that

(a) $Z \in \mathscr{E}$; (b) If $\mu$ is a measure and $w$ is the "round" version of $u$ as just defined, then $U \mu=W \mu$ if and only if $\mu(Z)=0$. Here, $U(x, d y)=u(x, y) \xi(d y)$, $W(x, d y)=w(x, y) \xi(d y)$, and $U \mu=\int U(x, y) \mu(d y), W \mu=\int W(x, y) \mu(d y)$.

In particular, it follows that $\xi(Z)=0$. This is a very important fact which motivates the introduction of the set $Z$. Using this fact, it follows (see [2]) that every excessive function $s$ which is not identically infinite can be written as

$$
s=h+\int u(x, y) m(d y)
$$

where $h$ is excessive and harmonic and $m$ is a Radon measure. Under the side condition $m(Z)=0$ the measure $m$ in (1) is unique; this $m$ will be called the Riesz measure of $s$. Also, one can show that for any Borel set $A, \int_{A} u(x, y) m(d y)$ is excessive and its Riesz measure is the restriction of $m$ to the set $A$.

DEFINITION. A nonnegative $\mathscr{E}$-measurable function $h$ will be called harmonic in an open set $D$, if for every compact set $K \subset D$ one has

$$
h(x)=P_{K^{c}} h(x) .
$$

When $D \equiv E$ we will say $h$ is a harmonic function.

Now we can show the following consequence of the results in [2].

THEOREM 1. For each $y$, the function $x \rightarrow u(x, y)$ is excessive and harmonic in the complement of the set $\{y\}$. In particular, the set $Z$ is empty and Radon measure $m$ in (1) is unique.

PROOF. It is shown in Proposition 3 of [2] that for every $y$, the function $x \rightarrow$ $u(x, y)$ is superaveraging. Let $\underline{u}$ denote its excessive regularization. Observe first that $\underline{u} \leq u$. On the other hand,

$$
\underline{u}(x, y)=\lim _{t \rightarrow 0} P u(x, y) \rightarrow u(x, y)
$$

because the function $x \rightarrow u(x, y)$ is lower semicontinuous for every $y$. Thus, $\underline{u}=u$.

Now let $D$ be an open set which is relatively compact. Then $P_{D} U f=U f$ for every nonnegative $f$ vanishing off $D$. It follows that for every $x, P_{D} u(x, y)=u(x, y)$ for $\xi$-almost all $y \in D$. Since paths of the process are continuous and $P_{D}(x, \cdot)$ is concentrated on $\partial D$ for $x \notin D$, and since $u$ is a continuous function, $y \rightarrow P_{D} u(x, y)$ is continuous on $D$ for each $x \in D^{c}$. Thus $P_{D} u=u$ on $D^{c} \times D$. Since $P_{D}(x, \cdot)=\varepsilon_{x}$ if $x \in D$,

$$
P_{D} u(x, y)=u(x, y), \quad(x, y) \in E \times D
$$

first, for relatively compact open $D$, and then for all open $D$ by an obvious approximation argument. Now by [5, Theorem 2, p. 373], it follows that the exceptional set $Z$ is empty; hence, by a previous remark the Radon measure $m$ occurring in (1) is unique. 
THEOREM 2. Let $s$ be an excessive function of $X$ which is not identically infinite, and whose Riesz measure is $m$. Then, $s$ is harmonic in an open set $D$ if and only if $m(D)=0$.

ProOF. Suppose $s$ is harmonic in $D$ and $s=h+U m$. Recall that $U m=$ $\int U(x, y) m(d y)$. Then, $U m$ is harmonic in $D$. We can assume $h=0$, namely, $s=U m$. For a Borel set $A$, write $s$ as $s_{A}=U m_{A}$, where $m_{A}$ is $m$ restricted to $A$. Then, one has $s=s_{D}+s_{D^{c}}$. Both terms on the right-hand side here are excessive and their sum is harmonic. Therefore, $s_{D}$ is harmonic in $D$. We want to show that $m_{D}=0$, i.e., $s_{D}=0$. For every compact set $K \subset D$,

$$
P_{K^{c}} s_{D}=s_{D} \text {. }
$$

The Riesz measure of the left-hand side in (2) cannot charge the interior of $K$. This follows from Theorem 2 of [2] and the argument used for Corollary 1 of [2]. In other words, the Riesz measure of $s_{D}$ which is $m$ restricted to $D$ cannot charge the interior of any compact set contained in $D$. Thus, $m(D)=0$.

Conversely, assume that the Riesz measure $m$ of $s$ does not charge $D$. Then, for any compact set $K \subset D, K^{c}$ is open and contains the support of $m$. Since $P_{D} u=u$ on $E \times D$ for all open $D$,

$$
P_{K^{c}} s=P_{K^{c}} U m=U m=s
$$

since the measure $m$ is carried by $D^{c} \subset K^{c}$, showing that $s$ is harmonic in $D$.

Now one can show the following

THEOREM 3. Let $h$ be excessive function of $X, h \not \equiv \infty$. If, in addition, $h$ is harmonic in an open set $D$, then $h$ is continuous in $D$.

PROOF. Without loss of generality we may assume that $D$ is a relatively compact open set. Observe that the function $s=P_{D} h$ is excessive. We claim it is harmonic in $D$. Indeed, if $K \subset D, K$ a compact set, then for $x \in K$ :

$$
P_{K^{c}} s(x)=P_{K^{c}} h(x)=s(x) .
$$

This follows from $s=h$ in $D$ and $X_{T_{K^{c}}} \in K, P^{x}$-a.s., by continuity of paths. Therefore, $s$ is harmonic in $D$. Now $s \equiv P_{D} h=U m$ where $m$ is carried by $\bar{D}$. But $s$ is harmonic in $D$ so that $m(D)=0$ by Theorem 2 . Thus $m$ is carried by $\partial D$ and we have

$$
P_{D} h(x)=\int_{\partial D} u(x, y) m(d y)
$$

The right-hand side of (3) is continuous by the assumption made about $u$. That proves the theorem since $h=P_{D} h$ in $D$.

THEOREM 4 (HARNACK INEQUALITY). Let $D$ be an open set and let $K$ be a compact subset of $D$. Then, there exists a finite constant $M>0$ depending on $D$ and $K$ only, such that for $x \in K, y \in K, h(x) \leq M h(y)$ for all excessive functions $h$ which are harmonic in $D$.

ProOF. Let $K \subset D_{0} \subset D$, where $D_{0}$ is a relatively compact open set. Put

$$
a=\inf _{x \in K, z \in \partial D_{0}} u(x, z), \quad b=\sup _{x \in K, z \in \partial D_{0}} u(x, z) .
$$


Then,

$$
h(x) \leq b m\left(\partial D_{0}\right) \leq \frac{b}{a} \int u(y, z) m(d z)=\frac{b}{a} h(y),
$$

where $m$ is the Riesz measure of $P_{D_{0}} h$. The theorem follows with $M=b / a$.

Finally, we have the following result.

THEOREM 5 (EQUI-CONTINUITY). Let $D$ be an open set and $x_{0} \in D$. Then, the set of excessive functions $\left\{h ; h\right.$ harmonic in $D$ and $\left.h\left(x_{0}\right) \leq 1\right\}$ is equi-continuous on every compact subset of $D$.

ProOF. Let $D_{0} \subset D$, where $D_{0}$ is a relatively compact open set. Then, for every excessive function $h$ which is harmonic in $D_{0}$, the Riesz measure of $P_{D_{0}} h$ is concentrated on $\partial D_{0}$ and has the total mass less or equal to $1 / a$ where $a=$ $\inf _{z \in \partial D_{0}} u\left(x_{0}, z\right)$. This fact is a consequence of (3). On the other hand, since $u$ is continuous in both variables and finite off the diagonal, the equi-continuity property is again a consequence of (3).

ACKNOWLEDGMENT. The author wishes to express his profound gratitude to Professor K. Murali Rao and the referee for valuable suggestions concerning this paper.

\section{REFERENCES}

1. R. M. Blumenthal and R. K. Getoor, Markov processes and potential theory, New York, Academic Press, New York, 1968.

2. K. L. Chung and M. Rao, A new setting for potential theory, Ann. Inst. Fourier (Grenoble) 30 (1980), 167-198.

3. K. L. Chung, Probabilistic approach in potential theory to the equilibrium problem, Ann. Inst. Fourier (Grenoble) 23 (1973), 313-322.

4. C. Constantinescu and A. Cornea, Potential theory on harmonic spaces, Springer-Verlag, Berlin and New York, 1972.

5. M. Liao, Riesz representation and duality of Markov processes, Seminaire de Probabilites XIX 1983/84, Lecture Notes in Math., vol. 1123, Springer-Verlag, Berlin and New York, 1985.

6. S. Port and C. Stone, Brownian motion and classical potential theory, Academic Press, New York, 1978.

7. Murali Rao, Brownian motion and classical potential theory, Lecture Notes Series \#67, Aarhus University, Denmark, 1977.

Department of Mathematics, University of Florida, Gainesville, Florida 32611 